\title{
Inequidad en la distribución de recursos humanos en los establecimientos del Ministerio de Salud de cuatro regiones del Perú
}

\author{
Inequity in the distribution of human resources for health at health care \\ services in four Peruvian regions
}

\author{
Betsy Moscoso Rojas ${ }^{1}$, Lizardo Huamán Angulo', Manuel Núñez Vergara1, \\ Ena Llamosas Felix ${ }^{1}$, Walter Perez \\ 'Dirección General de Gestión del Desarrollo de Recursos Humanos - Ministerio de Salud del Perú.
}

\begin{abstract}
Resumen
Introducción: La densidad de Recursos Humanos en Salud (RHUS) a nivel nacional en Perú, ha superado el umbral establecido por la OMS (25 por 10000 habitantes) para garantizar la cobertura de servicios de Salud. Sin embargo, este promedio nacional enmascara, a nivel regional, densidades inferiores al umbral estipulado, particularmente en las zonas más pobres del país. De ahí la necesidad de estudiar la posible existencia de inequidades en la distribución de RHUS a nivel regional, entendiendo a la inequidad como la innecesaria, evitable e injustificada carencia de RHUS. Objetivos: Determinar la inequidad en la distribución del personal de salud en los establecimientos de salud del Ministerio de Salud de cuatro regiones pobres del Perú (Cajamarca, Loreto, Apurimac y San Martín). Diseño: Estudio observacional descriptivo, de corte transversal. Institución: Dirección General de Gestión del Desarrollo de Recursos Humanos. Métodos: Se calculó la densidad de RHUS para cada región. Para la evaluación de la inequidad se calcularon los indices Gini, para el nivel departamental, y la T de Theil, para el nivel provincial. Resultados: La densidad de recursos humanos estuvo por debajo de mínimo estipulado por la OMS en 3 de las 4 regiones estudiadas. Los indices de Gini encontrados fueron 0,175 (Cajamarca), 0,157 (Loreto), 0,372 (Apurímac), y 0,146 (San Martín), que indican una tendencia a la equidad. En tanto, en el análisis intrarregional, los índices T de Theil muestran que un número importante de provincias tienen una buena distribución de RHUS. Conclusiones: A pesar de encontrar una baja densidad de RHUS en la mayoría de las regiones estudiadas, la distribución de los RHUS entre cada región muestra una tendencia hacia la equidad.
\end{abstract}

Palabras clave: Recursos humanos, equidad, Indicadores de Salud.

\section{Abstract}

Background: The density of Human Resources for Health $(\mathrm{HRH})$ at Peru has exceeded the threshold set by WHO to ensure coverage of health services, which is 25 per 10000 population. However this national average could masks regionally HRH densities that could be below the stipulated threshold, particularly in poorest areas. As a result it is necessary to study the existence of possible inequities in the distribution $\mathrm{HRH}$ inside the regions. Inequity must be understood as the unnecessary, avoidable, and unjustified lack of $\mathrm{HRH}$. Objectives: To determine inequities in the distribution of $\mathrm{HRH}$ at health care services of four poor regions of Peru (Cajamarca, Loreto, Apurimac and San Martin). Design: Cross sectional descriptive study. Institution: Dirección General de Gestión de Desarrollo de Recursos Humanos of Ministry of Health. Methods: Density of HRH for each region was calculated. In order to evaluate inequity, Gini index was calculated for the regional settings and Theil T Index for provincial settings. Results: HRH densities in 3 of the 4 regions were below the minimum stipulated by WHO. Gini index found were Cajamarca 0.175, Loreto 0.157, Apurimac 0.372 and San Martin 0.146 indicating a tendency to equity. Meanwhile, in intra-regional analysis, Theil T index showed that a significant number of provinces had good distribution of HRH. Conclusions: Despite low density of HRH in most regions studied, HRH distribution showed a trend towards equity.

Keywords: Human resources, equity, Health Status Indicators.

An Fac Med. 2015;76:35-40 / doi:10.15381/anales.v76i1.10968 


\section{INTRODUCCIÓN}

Los recursos humanos en salud (RHUS) constituyen el elemento esencial de los servicios de salud; la disponibilidad, distribución, calificación y compromiso de los mismos aseguran llegar a los objetivos sanitarios del sector salud porque garantizan la cobertura, impacto sanitario y satisfacción de los usuarios ${ }^{(1)}$. Cuantificar, caracterizar y evaluar la dotación, la distribución y la equidad de los recursos humanos es fundamental en cualquier esfuerzo de planificación. Por ello, planificar la oferta y la demanda de RHUS se convierte en un proceso indispensable de los gestores de salud, particularmente del nivel estratégico, y el contar con la información necesaria es una condición imprescindible ${ }^{(2,3)}$.

Para establecer si los recursos humanos son suficientes, no hay un estándar universalmente aceptado. Sin embargo, los países suelen compararse con otros dentro de una región o de un continente y utilizan algunos indicadores para expresar sus avances en términos de dotación. Ante la diversificación en el cálculo de indicadores, en el Informe Mundial sobre la Salud en el Mundo de $2006^{(4)}$, de la OMS y la Joint Learning Initiative (JLI) de la Fundación Rockefeller, se estimó que los países con una densidad menor a 2,28 médicos, enfermeras y parteras por cada 1000 habitantes generalmente no alcanzan la cobertura meta de $80 \%$ de partos atendidos por personas calificadas y la inmunización infantil. Esta meta crítica de aproximadamente 2,6 trabajadores por cada 1000 habitantes (para dejar lugar a la incertidumbre) fue calculada utilizando los mejores datos disponibles en los países miembros de la OMS de ese entonces, y se basó en el análisis del progreso entre y a través de las regiones hacia el logro de las tasas de cobertura meta para ciertas intervenciones de atención de la salud, establecidas de acuerdo a los Objetivos de Desarrollo del Milenio. En este informe, el Perú figuraba entre los países con déficit crítico de médicos, enfermeras y parteras. Sin embargo, a partir de esa fecha hasta hoy el Ministerio de Salud ha desarrollado importantes esfuerzos en disminuir las brechas de recursos humanos, privilegiando las zonas más pobres, de menor desarrollo y donde vive la población más excluida y vulnerable del país.

A partir de las estimaciones del Informe Mundial de Salud de 2006, la VII Reunión Regional de Observatorios de Recursos Humanos en Toronto (Canadá) estableció cinco desafíos en relación a las políticas de Recursos Humanos en los países de la región de la Américas. De ellos, el segundo desafío señala el compromiso de colocar a las personas adecuadas en los lugares adecuados, consiguiendo una distribución equitativa de los profesionales de salud en las diferentes regiones y de acuerdo con diferentes necesidades de salud de la población ${ }^{(5,6)}$. Dos años más tarde, todos los países de la región en el marco de reuniones organizadas por la OPS, firmaron las metas regionales para los RHUS 2007-2015. Se establecieron 20 metas a partir de los cinco desafíos planteados en Toronto. Esta agenda destacaba la necesidad de desarrollar el recurso humano para fortalecer la oferta en el primer nivel de atención ${ }^{(7)}$. Una de los metas establecidas señalaba que todos los países de la Región habrán logrado una razón de densidad de recursos humanos de 25 profesionales (médicos, enfermeras y parteras) por 10000 habitantes. Esta meta compromete a los países firmantes a desarrollar políticas para la adecuada dotación, distribución, captación y retención de recursos humanos. Otra de las metas señalaba que la brecha en la distribución de personal de salud entre zonas urbanas y rurales debería haberse reducido a la mitad en el 2015. Ello implica abordar el tema de las brechas de recursos humanos, tanto a nivel nacional como a nivel descentralizado en términos territoriales; con esta meta se busca disminuir la inequidad en la distribución de los recursos humanos. Además, se comprometían a establecer una unidad o dirección de recursos humanos para la salud responsable por el desarrollo de políticas y planes de recursos humanos ${ }^{(8)}$.

Si bien medir la disponibilidad de recursos humanos -empleando la densidad de RHUS- en los países es muy importante, evaluar su distribución y la inequidad que puede estar inherente a ella constituye un desafío tanto o más importante. Sobre todo si se considera que las cifras que se muestran como país, pueden esconder, al interior, diferencias significativas entre distintas áreas geográficas o territorios, constituyéndose así en inequidades entre grupos poblacionales, que no son percibidas cuando se muestra los promedios nacionales.

La inequidad en salud describe la desigualdad innecesaria, evitable e injustificada en el estado de salud y el acceso a servicios por parte de una población. En el campo de los RHUS significa el acceso a personal de salud calificado, que brinde atención a sus necesidades de salud. Es decir, equidad no significa tener igual cantidad de recursos humanos en los ámbitos geográficos, sino ausencia de diferencias evitables en las oportunidades de contar con recursos humanos que atiendan la salud cuando se necesite. El análisis de la densidad de RHUS ha sido usado como indicador para evaluar la inequidad en regiones como China, India ${ }^{(9)}$, Brasil ${ }^{(10)}$ y Chile ${ }^{(11)}$. Este tipo de análisis se sustenta en que la distribución de RHUS a nivel mundial no es homogénea. En muchos casos, además tiene implícita una connotación económica. Es el caso de Canadá y Estados Unidos, que pese a tener un $10 \%$ de morbilidad mundial, concentran el 37\% de fuerza de trabajo sanitario mundial y más del $50 \%$ de los recursos financieros mundiales destinados a la salud. Realidad que contrata con la observada en África, en donde se concentra más de $24 \%$ de la carga de morbilidad, pero solo $3 \%$ de los trabajadores sanitarios y menos del $1 \%$ de los recursos financieros mundiales ${ }^{(12,13)}$. En Perú, para el año 2007, existían brechas de recursos humanos en varias regiones, siendo Cajamarca, Loreto y Piura donde se hallaba el mayor déficit ${ }^{(14)}$. 
La inequidad es un problema que se encuentra presente en mayor o menor medida en las sociedades con desigual distribución de la riqueza y los indicadores que la miden pueden brindarnos una importante aproximación al nivel de justicia social que la sociedad ha alcanzado ${ }^{(15)}$. Por lo tanto, su estudio en el campo de los recursos humanos resulta de vital importancia para generar evidencias que contribuyan a mejorar el acceso equitativo de la población a recursos humanos calificados, para atender su salud y disminuir sus riesgos de enfermar o morir por problemas de salud.

Es así que el objetivo del presente estudio fue determinar la inequidad derivada de la distribución del personal de salud, en base a la densidad y brecha de profesionales de la salud (médicos, enfermeras y obstetrices) en los establecimientos de salud del Ministerio de Salud de cuatro regiones pobres, y con población vulnerable, del Perú (Cajamarca, Loreto, Apurímac y San Martín), con la finalidad de diseñar políticas y estrategias que conduzcan a la equidad en la distribución de los recursos humanos en estas regiones y que el estudio se constituya en un referente para realizar otros estudios en las demás regiones del país y para perfeccionar la metodología de la medición.

\section{MÉTODOS}

En octubre de 2013, la Dirección General de Gestión del Desarrollo de Recursos Humanos realizó un estudio observacional, descriptivo, de corte transversal.

Como población de estudio, se incluyó a todos los médicos, enfermeras y obstetrices que desempeñaban actividades asistenciales y administrativas en los establecimientos del Ministerio de Salud del Perú (MINSA) pertenecientes a las Direcciones Regionales de Salud (DIRESAs) y Gobiernos Regionales (GR) de Cajamarca, Loreto, Apurímac y San Martín. Es así que se incluyó información de 801 estableci- mientos de salud de Cajamarca, 365 establecimientos de salud de Loreto, 233 establecimientos de salud de Apurímac y 375 establecimientos de salud de San Martin.

La información del personal de salud, así como la de los establecimientos de salud, fue obtenida del Registro Nacional de Personal de Salud (INFORHUS) del MINSA, durante el mes de octubre 2013. La información se organizó en dos niveles: departamental y provincial.

La densidad de RHUS se determinó siguiendo la formula determinada por la OPS ${ }^{(4)}$, en la cual el numerador lo constituyó el número de médicos, enfermeras y obstetrices de los establecimientos de salud incluidos, y el denominador, la estimación de número de habitantes de las cuatro regiones estudiadas que debe atenderse en los establecimientos incluidos. Para esta estimación se tomó en cuenta el número de habitantes que no tenían acceso a ningún de seguro de salud.

Para el análisis de la inequidad se calcularon los índices de Gini para evaluar la inequidad a nivel regional y la T de Theil para evaluar la inequidad entre las provincias de cada región. Estos indicadores han sido empleados ya para evaluar las inequidades en la distribución de los RHUS ${ }^{(9,10,16)}$. El coeficiente de Gini constituye un indicador del grado de asimetría en la distribución de una variable; así, mide la desigualdad de la distribución de los recursos humanos. Asume valores entre 0 y 1 , donde cero implica perfecta igualdad en la distribución y uno, perfecta desigualdad. La evaluación del índice de Gini además implica la construcción de la curva de Lorenz, que grafica la distribución relativa de una variable en un dominio determinado. Así, el área de la superficie entre la curva y la diagonal (área de concentración), cuando más pequeña sea, más equitativa será la distribución. Por su parte el índice T de Theil permite analizar, describir y descomponer las inequidades en la distribución de los recursos humanos, considerando un factor que contribuye a la inequidad existente entre las provincias de cada departamento. Asume valores entre 0 y 1 ; cuanto más cercano sea el valor a 1 , peor será la distribución del RHUS.

\section{RESULTADOS}

\section{Región Cajamarca}

En Cajamarca, la densidad de RHUS encontrada fue de 13,2 por 10000 habitantes, que está muy por debajo del mínimo señalado por la OPS. Por provincias, se observó que las densidades de RHUS más baja se encontraba en Celendín, Hualgayoc y San Ignacio con 12,3, 14,5 y 15,5 RHUS por 10000 habitantes, respectivamente. La evaluación de la inequidad de la distribución de RHUS a nivel regional mostró que el índice de Gini para Cajamarca fue de 0,175 (figura 1), que es un valor cercano a la línea de equidad. En tanto que en el análisis intrarregional se encontró que los índices $\mathrm{T}$ de Theil mostraban que las provincias de Contumaz, Jaén y Santa Cruz mostraban una peor distribución de RHUS. De otro lado, las provincias donde la distribución de RHUS fue más equitativa fueron las provincias de Hualgayoc, Cajabamba y San Pablo (figura 2).

\section{Región Loreto}

La densidad de recursos humanos en la Región Loreto fue de 13 x 10000 habitantes, también por debajo de punto corte establecido por la OMS. El cálculo del Coeficiente de Gini para la región Loreto fue 0,157 , el cual nos indica que hay una tendencia a la equidad (figura 3). El índice $T$ de Theil mostró que en las provincias de Loreto existe una buena distribución del RHUS ( $T$ de Theil $<0,4$ en todos los casos), y por tanto menor inequidad, siendo la provincia de Loreto la que presentó la mejor distribución (figura 4).

\section{Región Apurímac}

En Apurímac, la densidad de recursos humanos fue de 34,8 recursos humanos 


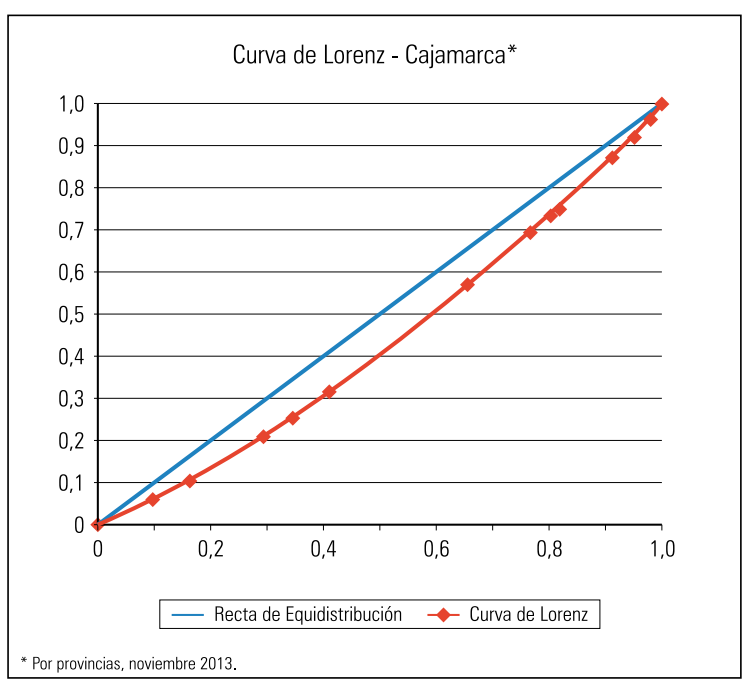

Figura 1. Curva de Lorenz e índice de Gini, Región Cajamarca.

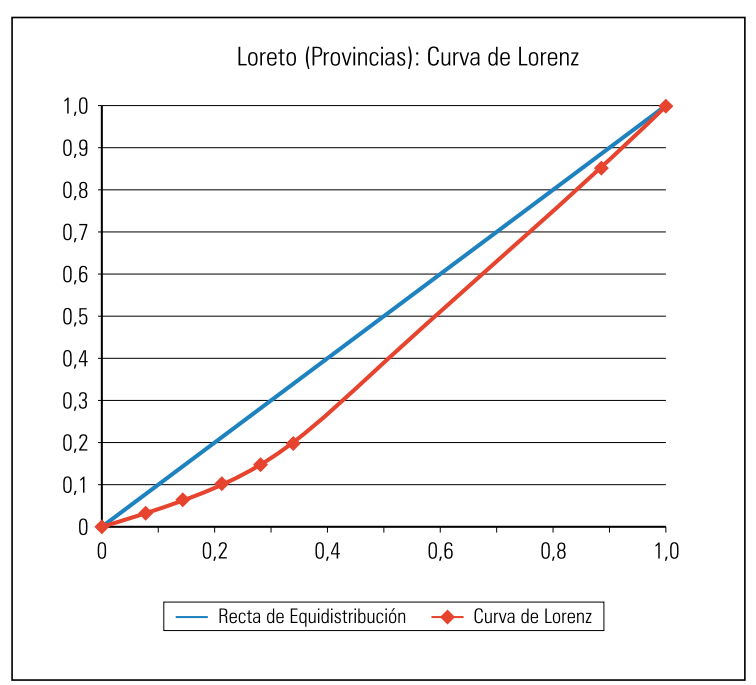

Figura 3. Curva de Lorenz e índice de Gini, Región Loreto.

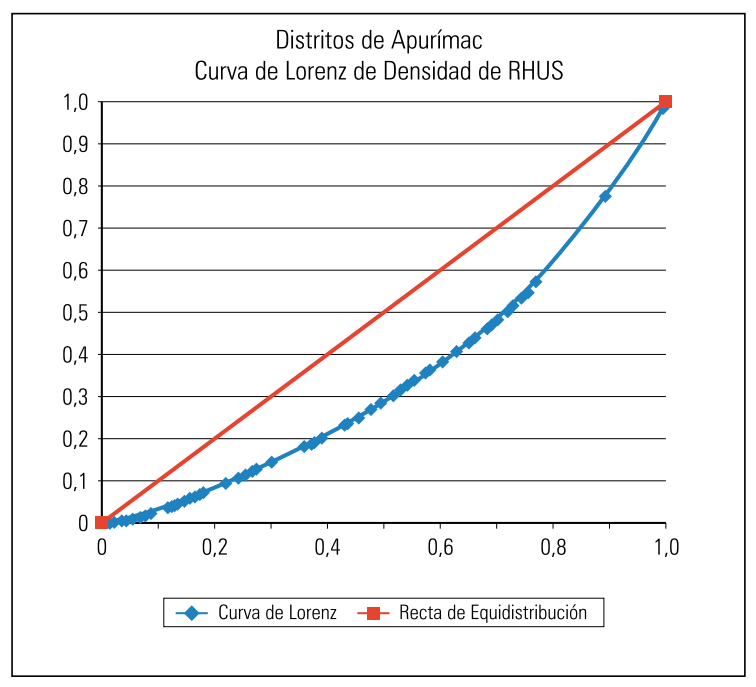

Figura 5. Curva de Lorenz e índice de Gini, Región Apurímac.

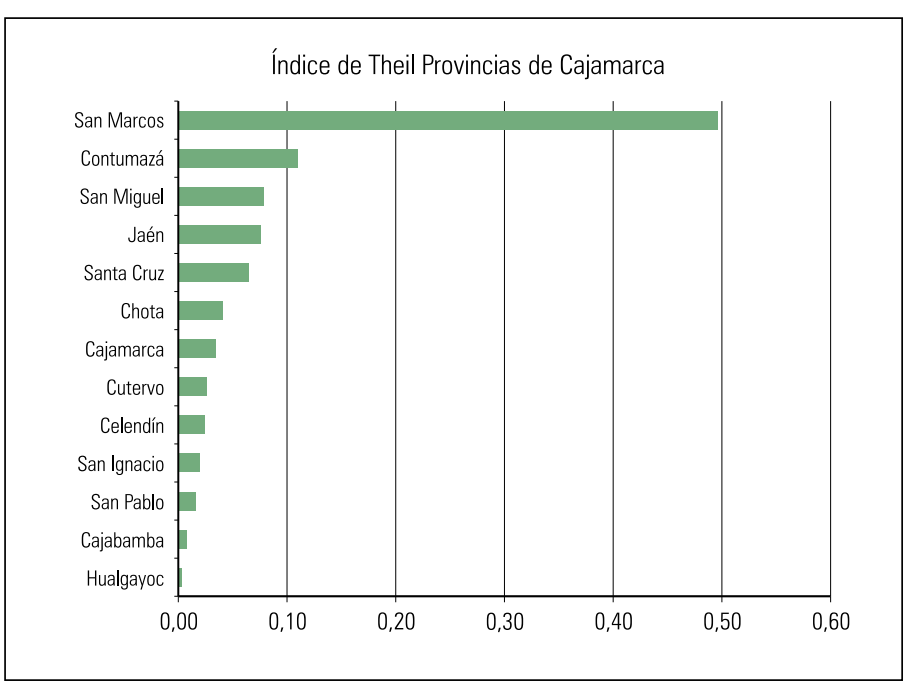

Figura 2. Índice de Theil, Región Cajamarca.

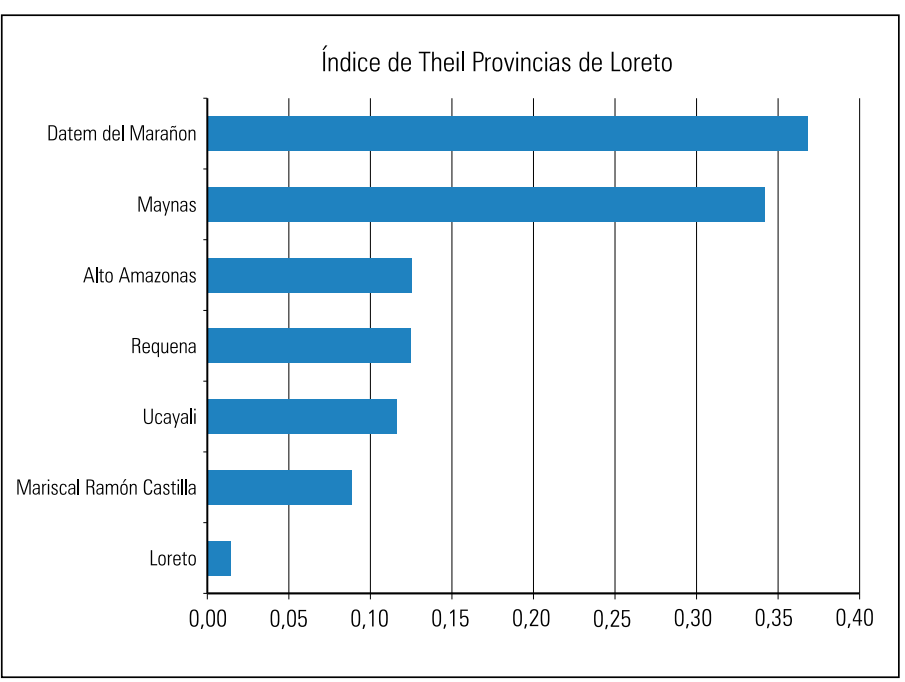

Figura 4. İndice de Theil, Región Loreto.

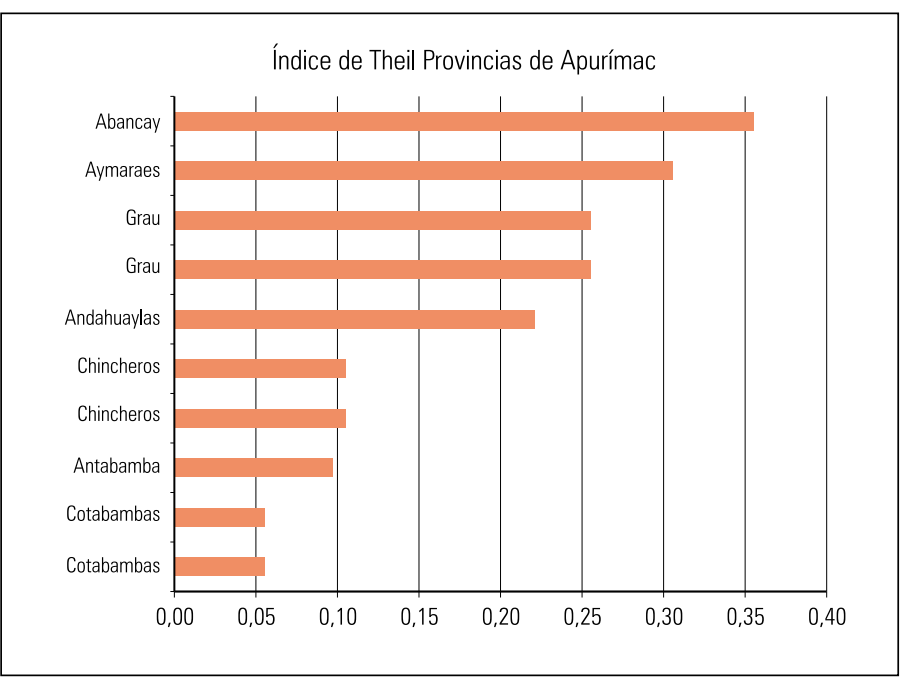

Figura 6. Índice de Theil, Región Apurímac. 


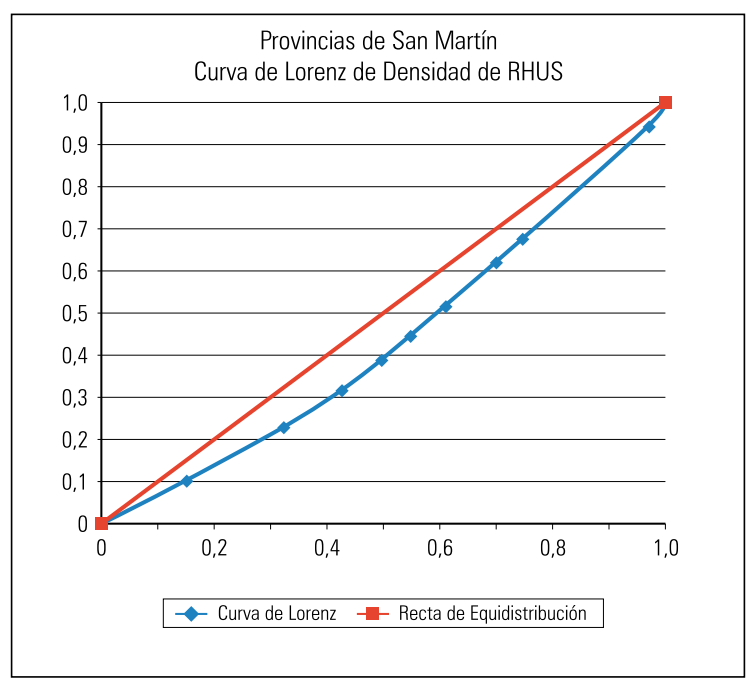

Figura 7. Curva de Lorenz e índice de Gini, Región San Martín.

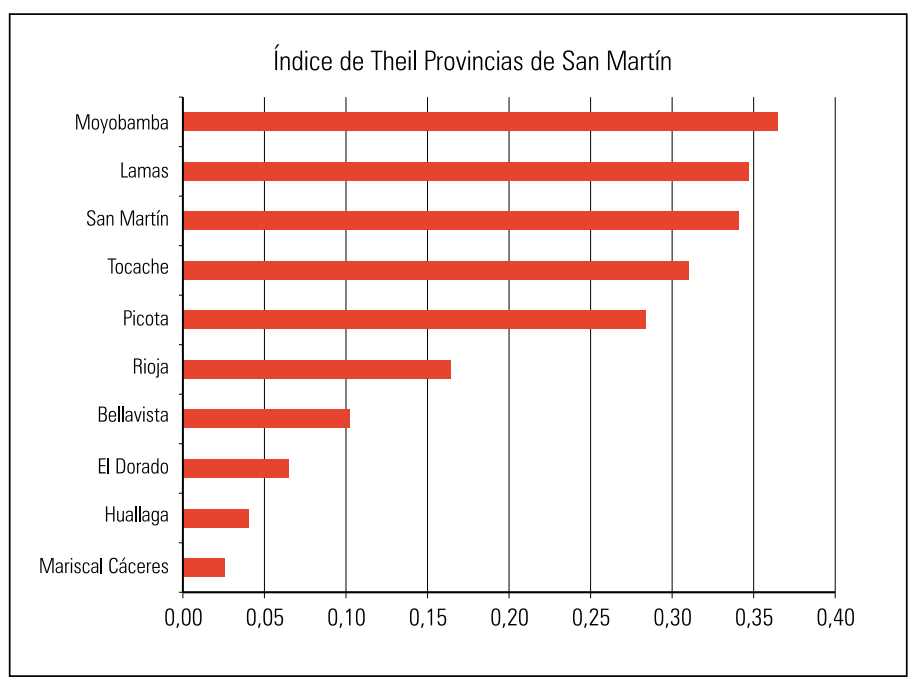

Figura 8. İndice de Theil, Región San Martín. por 10000 habitantes, constituyéndose en la única región que se encuentra por el umbral determinado por la OMS. El cálculo del Coeficiente de Gini a nivel provincial fue 0,372 (figura 5). Los resultados del cálculo de los índices Theil para las provincias de la región se muestran en la figura 6; en las provincias de Apurímac existió una buena distribución del RHUS (T de Theil < 0,4 en todos los casos), y por tanto menor inequidad.

\section{Región San Martín}

La densidad de recursos humanos en San Martín fue 11,7 recursos humanos por 10000 habitantes. El cálculo del Coeficiente de Gini a nivel provincial fue 0,146 (figuras 7). Los resultados del cálculo de los índices Theil para las provincias de la región muestran que en las provincias de San Martin existió una buena distribución del RHUS (T de Theil $<0,4$ en todos los casos), y por tanto menor inequidad (figura 8).

\section{DISCUSIÓN}

Los países firmantes del "Llamado a la acción de Toronto" se comprometieron a velar por la distribución de recursos humanos de manera adecuada, eficiente, equitativa y oportuna, con la finalidad de mejorar el acceso a de la población a los servicios de salud, y así mejorar sus estándares de salud ${ }^{(17)}$. En ese contexto se requiere contar con profesionales de la salud en número suficiente, con las competencias adecuadas y comprometidos con el logro de resultados sanitarios en todos los niveles de complejidad del sistema. En nuestro estudio se encontró que la densidad de recursos humanos en tres de las cuatro regiones estudiadas muestra un grave déficit, puesto que se encuentran por debajo del corte mínimo de $25 \times 10000$ habitantes establecido por la OMS como el límite necesario para garantizar la prestación de servicios esenciales. Este déficit podría estar comprometiendo el logro de los resultados sanitarios y la capacidad de respuesta del sistema sanitario regional. En Perú, estudios previos ya habían descrito un déficit de médicos, particularmente en el primer nivel de atención; y una inadecuada distribución de ellos, pudiéndose observar concentraciones mayores en las zonas de mayores recursos; por lo que se concluye que esta inadecuada distribución no solo es geográfica sino que es también socio-económica ${ }^{(7)}$.

Antes de iniciado el estudio, se pensaba encontrar un alto grado de inequidad en la distribución de los recursos humanos en las regiones estudiadas.
Los resultados, sin embargo, muestran que la inequidad existente en la distribución de recursos humanos en estas regiones es baja. Pese a existir un déficit marcado de recursos humanos, los resultados del análisis de equidad muestran que, de manera general, la distribución de recursos humanos por provincias tiene mayor tendencia a la equidad. Es posible que esto se deba a los esfuerzos que en los últimos años ha desarrollado el MINSA en redistribuir las plazas de los profesionales que realizan el servicio rural urbano marginal (SERUMS) hacia las zonas más pobres, de menor desarrollo social y donde vive la población más excluida y vulnerable, considerando además los lugares con menor densidad de recursos humanos. Además, otras iniciativas implementadas por los gobiernos regionales y locales en las cuales se ha priorizado la dotación de recursos humanos han complementado los esfuerzos realizados por el Ministerio de salud y contribuido así con esta tendencia a la equidad.

Es necesario resaltar el valor que tienen, para el estudio de la inequidad, los índices de Gini y de Theil. Estas son herramientas metodológicas que permiten identificar las inequidades, no solo en el campo de la economía sino aplicadas también al campo de los RHUS. Se debe considerar que, en conjunto, es- 
tos índices nos permiten identificar las deficiencias en la asignación de los recursos humanos y las concentraciones de recursos humanos en determinadas áreas, de manera que podamos reorientar la asignación en función de las necesidades y en el marco de la equidad en el acceso a atención a través de los recursos humanos.

Algunas limitaciones deben ser declaradas. La primera de ellas se encuentra relacionada al indicador de densidad de RHUS. Este indicador fue desarrollado en 2006, debido a las características de las atenciones de salud priorizadas que se tenían en ese momento; solo incluyen a los tres grupos profesionales incluidos en este estudio, dejando por fuera a otros grupos profesionales; por tanto, dejan por fuera el rango completo de servicios preventivos, promocionales, curativos y de rehabilitación, así como actividades de gerencia y de apoyo de los sistemas de salud que proporcionan el RHUS. En contraste, las fortalezas del estudio radican en el diseño metodológico, el cual ha permitido ahondar en el análisis de la inequidad en la distribución de RHUS en cuatro regiones pobres del Perú. Metodologías de esta naturaleza permiten identificar inequidades tanto entre regiones -gracias a la inclusión del índice de Gini- como dentro de las mimas, gracias al uso del índice de Theil. Ello permite conocer de manera adecuada las regiones y provincias en donde son necesarias intervenciones, haciendo que estas sean más eficaces y eficientes.

Concluimos que en el presente estudio se encontró una baja densidad de RHUS en tres de las cuatro regiones estudiadas (Cajamarca, Loreto y San Martín). La baja densidad de RHUS en estas regiones podría estar comprometiendo el logro de los objetivos sanitarios dentro de ellas. A pesar del déficit en el número de RHUS observados en cada región, el análisis de la equidad de la distribución de los RHUS al interior muestra tendencia a la equidad.

Existe la necesidad de desarrollar estudios en las otras regiones del país que complementen el presente estudio, que no solo generen datos e información, sino que en su ejecución permitan desarrollar acciones de coordinación, asistencia técnica y construcción de políticas conjuntas con nivel regional y local, que puede constituirse en un factor dinamizador que potencie la relación a favor de logros y la misión institucional como sector salud.

\section{REFERENCIAS BIBLIOGRÁFICAS}

1. Brito P. Impacto de las reformas del sector de la salud sobre los recursos humanos y la gestión laboral. Rev Panam Salud Publica. 2000; 8 (1-2): 43-54. doi: dx.doi.org/10.1590/S102049892000000700008

2. Nigenda G, Ruiz J, Wirtz V, Gonzáles M, Gonzáles M, Bejarano R, Aguilar E. Formación, empleo y regulación de los recursos humanos para la salud: Bases para su planeación estratégica. Cuernavaca: Instituto Nacional de Salud Pública, 2010.

3. Rovere M. Planificación estratégica de recursos humanos en salud. Serie Desarrollo de Recursos Humanos. Washington D.C.: Organización Panamericana de la Salud, 1993.

4. Organización Mundial de la Salud. Informe sobre la salud en el mundo 2006: Colaboremos por la salud. 1 ra edición. Ginebra: Organización Mundial de la Salud; 2006.

5. Organización Panamericana de la Salud. Llamado a la Acción de Toronto. 2006 - 2015: Hacia una década de Recursos Humanos en Salud para las Américas. Reunión Regional de los Observatorios de Recursos Humanos en Salud, Toronto, Canadá; 4-7 de octubre de 2005. Disponible en: http:// www.observatoriorh.org/sites/default/files/webfiles/ fulltext/OPS_desafios_toronto_2005.pdf. Acesso: 7 de diciembre de 2014

6. Organización Panamericana de la Salud. Metas regionales en materia de recursos humanos para la salud 2007-2015. Resolución Nro. CSP27/10. Washington D.C., 2007.

7. Núñez M. Estudio de la dotación de profesionales de la salud en los establecimientos del Ministerio de Salud. Lima: Ministerio de Salud del Perú, 2007.

8. Organización Panamericana de la Salud. Manual de Medición y Monitoreo de indicadores de las metas regionales de recursos humanos para la salud: un compromiso compartido. $1^{\text {a }}$. ed. Washington: OPS; 2011.
9. World Health Organization. Measuring health workforce inequalities: methods and application to China and India. Ginebra: World Health Organization, 2010.

10. Sousa A, Dal Poz M, Carvalho C. Monitoring Inequalities in the Health Workforce: The Case Study of Brazil 1991-2005. PLoS ONE. 2012;7(3):e33399. doi: 10.1371/journal.pone.0033399

11. Banco Mundial - Gobierno de Chile. Estudio de Brechas de Oferta y Demanda de Médicos Especialistas en Chile. Santiago: Subsecretaria de Redes Asistenciales Departamento de Estudios de Recursos Humanos, 2010.

12. Chen L, Evans T, Anand S, Boufford JI, Brown H, Chowdhury $\mathrm{M}$, et al. Human resources for health: overcoming the crisis. Lancet. 2004;364:1984-90

13. Speybroeck N, Ebener S, Sousa A, Paraje G, Evans $\mathrm{DB}$, Prasad A. Inequality in access to human resources for health: measurement issues. Ginebra: Organización Mundial de la Salud, 2006. Fecha de acceso 20 de mayo 2014. (Disponible en: http:// www.who.int/hrh/documents/en/)

14. Carrasco V, Lozano E, Velásquez E. Análisis actual y prospectivo de la oferta y demanda de médicos en el Perú 2005-2011. Acta méd peruana. 2008;25(1):22-9.

15. Zevallos L, Pastor R, Moscoso B. Oferta y demanda de médicos especialistas en los establecimientos de salud del Ministerio de Salud: brechas a nivel nacional, por regiones y tipo de especialidad. Rev peru. med exp salud publica. 2001;28(2):177-85.

16. Schneider MC, Castillo-Salgado C, Bacallao J, Loyola E, Mujica OJ, Vidaurre M y col. Métodos de medición de las desigualdades de salud. Rev Panam Salud Publica. 2002;12(6):398-414.

17. López C. La medición del estado de salud de la población y su relación con los determinantes. Rev Cubana Salud Pública. 2007;33(1):75-9.

\section{Agradecimientos:}

Al equipo de CARE Perú y al Fondo de Población de las Naciones Unidas en Perú (UNFPA-Perú) por el apoyo brindado al Ministerio de Salud en la ejecución y financiamiento del estudio. A los equipos de Gestión de los Gobiernos Regionales y a los equipos de las Gerencias de Desarrollo Social de las regiones estudias por las facilidades brindadas para la ejecución del estudio. Al equipo del Observatorio Nacional de Recursos Humanos del MINSA y al equipo del Observatorio de Cajamarca por el apoyo durante la recolección y análisis de la información.

Conflictos de interés:

Los autores declaran no tener conflictos de interés con la publicación del artículo.

Correspondencia:

Betsy Moscoso

bmoscoso2003@yahoo.com 